Journal of the Mathematical Society of Japan Vol 1, No. 4, April, 1950.

\title{
On removable singularities of an analytic function of several complex variables.
}

\author{
Masatsugu Tsuji.
}

(Received June. 18, 1947)

1. Let $u(P)=u\left(x_{1}, \ldots \ldots, \ldots, x_{n}\right)$ be defined in a domain $D$ in an $n$-dimensional space and all its partial dervatives of the second order be continuous and satisfy the equation:

$$
\Delta u=\frac{\partial^{2} u}{\partial x_{1}^{2}}+\cdots+\frac{\partial^{2} u}{\partial x_{n}^{2}}=0
$$

then $u(P)$ is called a harmonic function in $D$. It is easily seen that $u(P)=\widehat{O P^{n-2}}(n \geq 3)$ is a harmonic function, where $P$ is a variable point and $O$ is a fixed point.

Let $\Sigma$ be a sp'ere in an $n$-dimensional space with $O$ as its center and $R$ be its radius and $S$ be its boundary. Let $Q$ be a point of $S$ and $\varphi(Q)$ be an integrable function on $S$. We define a Poisson integial with $\varphi(Q)$ as its boundary value : ${ }^{1)}$

$$
u(P)=\frac{1}{R S_{n}} \int_{S} \varphi(Q) \frac{R^{2}-\overline{O P}^{2}}{\overline{P O^{n}}} d \sigma_{\iota},
$$

where $S_{n}$ is the surface area of a unit sphere and $d \sigma_{Q}$ is the surface element of $S$ at $Q$. Then $u(P)$ is harmonic in $\Sigma$.

We can prove that $u(P)$ tends to $\varphi(Q)$ almost everywhere on $S$, when $P$ tends to $Q$ non-tangentially to $S$. If $\varphi(Q)$ is continuous at $Q_{0}$, then $u(P)$ tends to $\varphi\left(Q_{0}\right)$, when $P$ tends to $Q_{0}$ from the inside of $\Sigma$. Let $u(P)$ be a bounded harmonic function in $\Sigma$, then $\lim u(P)=\varphi(Q)$ exists almost everywhere on $S$, when $P$ tends to $Q$ non-tangentially to $S$ and $u(P)$ can be expressed by $(2)^{2}$.

1) For an n-dimensional Poisson integral, c. f. C. Carathéodory: On Dirichlet problem. Amer. Jour. Math. 59(193\%).

2) The case $n=2$ is the well known theorems of Fatou and Schwarz. For the case $n=3$ I have proved analogous theorems in a paper: On Fatou's theorem on Poisson integrals. Jap. Jour-Math. 15(1938). The method can be applied for the general case. 
We will prove:

Lemma 1. Let $\sum$ be a sphere of radius $R$ with $O$ as its center and $\sigma$ be a concentric sphere of radius $\rho<R$. If $u(P)$ is harmonic in $\Sigma$ and $u(P) \equiv \mathrm{O}$ in $\sigma$, then $u(P) \equiv \mathrm{o}$ in $\sum$.

Proof. $u(P)$ is expressed by a Poisson ingearal:

$$
u(P)=\frac{1}{R, S_{n}} \int_{S_{1}} u(Q) \frac{R_{1}^{2}-\overline{O P^{2}}}{\overline{P Q^{n}}} d \sigma_{Q},
$$

here $S_{1}$ is the boundary of a concentric sphere $\Sigma_{1}$ of radius $R_{1}<R$.

If we put $r=\overline{O P}$ and $\theta$ be the angle between $O P$ and $O Q$, then $\bar{P} Q^{2}=$ $R_{1}^{2}-2 R_{1} r \cos \theta+r^{2}$, which vanishes for $r=R_{1} e^{ \pm i \theta}$, so that if we consider $r$ a complex variable,

$$
\frac{R_{1}^{2}-\overline{O P^{2}}}{\overline{P Q^{n}}}=\frac{R_{1}^{2}-r^{2}}{\left(R_{1}^{2}-2 R_{1} r \cos \theta+r^{2}\right)^{\frac{n}{2}}}
$$

is a regular function of $r$ for $|r|<R$. Let $Q_{0}$ be a point on $S_{1}$ and $P$ vary on the segment $O Q_{0}$, then $u(P)$ is a regular function of $r$ for $|r|<R_{1}$, which, by the hypothesis, vanishes for $\mathrm{o} \leqq r \leqq \rho$, hence $u(P)=\mathrm{o}$ for $\mathrm{o} \leqq r \leqq R_{1}$, so that $u(P)$ vanishes on the segment $O Q_{0}$ and since $Q_{v}$ is arbitrary, $u(P) \equiv \mathrm{o}$ in $\Sigma_{1}$. Hence for $R_{1} \rightarrow R$, we have $u(p) \equiv \mathrm{o}$ in $\Sigma_{1}$ q. e. d:

From this we can deduce the following

Lemma 2 Let $u(P)$ be halmonic in a domain $D$ and if $u(P) \equiv 0$ in a partial domain $D_{1} \subset D$, then $u(P) \equiv \mathrm{O}$ in $D$.

Theorem I Let $\Sigma$ be a sphere and $E$ be a closed set in $\Sigma$. Then in general $\Sigma-E$ consists of at most countable number of componints $D_{i}$. Suppose that there exists in each componet $D_{i}$ a posituve harmonnc function $v_{i}(P)$, such that lim $v_{i}(P)=+\infty$, when $P$ tends to any boundary point of $D_{i}$, which belongs to $E$, then

(i) $\Sigma-E$ is connected, so that it consists of only one component.

(ii) Lot $S_{E}$ be the parı of $E$, which lies on the boundany $S$ of $\Sigma$, then $S_{E}$ is of surface measure zero. Hence by Fubini'i theorem, $E$ is of measure zero, so thai $E$ has no inner points.

Proof. (i) Suppose that $\Sigma-E$ is not connected and consists of more than one component and let $D_{1}, D_{2}$ be any two components. The boundary point of $D_{1}$ belongs to $S$ or $E$. If all its boundary points belong to $E$, then $v_{1}(P) \equiv+\infty$ in $D_{1}$, which is absurd. Hence $D_{1}$ has a boundary point on $S$, so that if we denote the part of the boundary of $D_{1}$, which lies on $S$ by $S_{1}$, then $S_{1}$ has an inner point on $S$. Similarly the part $S_{2}$ 
of the boundary of $D_{2}$ on $S$ has an inner point on $S$.

We put

$$
u_{1}(P)=\frac{1}{R S_{n}} \int_{S_{2}} \frac{R^{2}-\overline{O P^{2}}}{\overline{P Q^{n}}} d \sigma_{Q}
$$

then $u_{1}(P)$ is a bounded harmonic function in $\Sigma$ and $u_{1}(P)=0$ on $S_{1}$. Let for any $\varepsilon>0$,

$$
\Phi_{\varepsilon}(P)=u_{1}(P)-\varepsilon v_{1}(P)
$$

then since $v_{1}(P)_{0} \geqq 0$, we have $\Phi_{\varepsilon}(P) \leqq 0$ on $S_{1}$ and since $u_{1}(P)$ is bounded and $\lim v_{1}(P)=+\infty$ on $E$, we have $\Phi_{\varepsilon}(P) \bar{\sum}$ on $E$, so that by the maxim $\lrcorner m$ principle, $\Phi_{\varepsilon}(\rho) \leqq 0$ in $D_{1}$, hence for $\varepsilon \rightarrow 0, u_{1}(P) \leqq 0$ il $D_{1}$.

S imiarly considering $u_{1}(P)+\varepsilon v_{1}(P)$, we have $u_{1}(P) \geqq 0$ in $D_{1}$, so that $u_{1}(P) \equiv \mathrm{o}$ in $D_{1}$, hence by Lemma $2 u_{1}(P) \equiv \mathrm{o}$ in $\Sigma$, which is absurd, since $u_{1}(P)$ tends to 1 , when $P$ tends to an inner point of $S_{2}$. Hence $\Sigma-E$ is connected.

(ii) Suppose that $S_{F}$ is of positive surface measure and put

$$
u(P)=\frac{1}{R S_{n}} \int_{S_{F}} \frac{R^{2}-\overline{O P^{2}}}{\overline{P Q^{n}}} d \sigma_{Q},
$$

then $u(P)$ is a bounded harmonic function in $\Sigma$ and tends to 1 almost everywhere on $S_{F}$, when $P$ tends to $S_{F}$ non-tangentially to $S$. But from the argument in (i), we see that $u(P) \equiv 0$ in $\sum$, which is a contradiction. Hence $S_{E}$ is of surface measure zero. Hence by Fubini's theorem, $E$ is of measure zero. q.e.d.

Theorem 2. Let $E$ bi a clusid set $2 n$ an $n$-dimerisional space and $D$ be its nigibourh od. Suppos" that there exists a positive ha monic function $v(P)$ in $D-E$, such that lim $v(P)=+\infty$, when $P$ tendes to any point of $E$. Let $u(P)$ be a bounded harmonic function in $D-E$, then $u(P)$ is harmonic on $E$.

Proof. Let $O$ be a point of $E$ and $\Sigma$ be a sphere about $O$ of radius $R$, which is contained in $D$. Then by Theorem $1, \Sigma-E$ is connected and $E$ has no inner points. We coustruct a Poisson integral witil $u(Q)$ as its boundary value :

$$
u_{1}(\rho)=\frac{1}{R S_{n}} \int_{S-S_{F}} u(Q) \frac{R^{2}-\overline{O P^{2}}}{\overline{P Q^{n}}} d \sigma_{Q}
$$


where $S_{E}$ is the part of $E$, which lies on the bo indary $S$ of $\Sigma$. Then $u_{1}(P)$ is a bounded harmonic function in $\sum$ and $u_{1}(P)=u(P)$ on $S-S_{k}$, so that $U(P)=u(P)-u_{1}(P)$ is a bounded ha monic function in $\Sigma-E$, which vanishes on $S-S_{E}$. Hence by the argument of Thecrem 1 we have $U(P) \equiv 0$ in $\Sigma-E$, or $u(P)=u_{1}(P)$ in $\Sigma-E$. Since $E$ has no inner points, we can continue $u(P)$ has monically in $\Sigma$ by $u(P)=u_{1}(P)$, so that $u(P)$ is harmonic on $E$. q.e.d.

As an application of Theorem 2, we have

Theorem 3. Let $g\left(z_{1} \ldots \ldots \ldots z_{n}\right)$ be a regular function of $n$ complex variabls in a 2 -dimensional domain $D$ and $E$ be the manifold defined by $g\left(s_{1}, \ldots \ldots \ldots, \ldots, z_{n}\right)=0$. Let $f\left(z_{1}, \ldots \ldots \ldots, z_{n}\right)$ be a bounded regular frinction in $D-E$, then $f\left(z_{1}, \ldots \ldots, \ldots, z_{n}\right)$ is regular on $E .{ }^{3)}$

Proof. It is evident that $E$ has no inner roints. If we put $f=u+i v$, then $u, v$ are bounded harmonic functions in $D-E$. Let $\left|g\left(z_{1}, \ldots \ldots \ldots, z_{n}\right)\right|$ $\leqq M$ in $D$, then

$$
V^{\prime}(P)=\log \frac{M}{|g(P)|,} \quad P=\left(z_{1}, \ldots \ldots \ldots, z_{n}\right)
$$

is a positive harmonic function in $D-E$, such that $\lim V(P)=+\infty$, when $P$ tends to any point of $E$, hence by Theorem $2, u$ and $v$ are harmonic on $E$, so that $f$ is regular on $E$. q.e.d

Fiom Theorem 1, we have

Theorem 4. Let $g\left(z_{1}, \ldots \ldots . ., z_{n}\right)$ be regular in a clomain $D$ and $E$ be the manifold defined by $g\left(z_{1}, \ldots . . . ., z_{n}\right)=0$ and $\sum$ be a sphere which is con ained in $D$ and contains points of $E$, then $\Sigma-E$ is connected ${ }^{4)}$ and $S_{E}$ is of surface $m$ asure zero, where $S_{E}$ is the part of $E$, which lics on the boundary $S$ of $\Sigma$.

2. Let $E$ be an $(n-2)$-dimensional manifold in an $n$-dimensional $\left(x_{1}, \ldots \ldots \ldots, x_{n}\right)$-space, which is defined by

$$
x_{i}=\varphi_{i}\left(t_{1}, \ldots \ldots, \ldots, t_{n-2}\right)=\varphi_{i}(\dot{t}) \quad(i=1,2, \ldots \ldots, n),
$$

where $\varphi_{i}(t)$ are defined in an $(n-2)$-dimensional domain $\Delta$ in $\left(t_{1}, \ldots . . t_{n-2}\right)$ -space and satisfy the Lipschitz's condition:

$$
\left|\varphi_{i}(t)-\varphi_{i}\left(t^{\prime}\right)\right|=\left|\varphi\left(i_{1}, \ldots \ldots, t_{n-2}\right)-\varphi_{i}\left(t_{1}^{\prime}, \ldots \ldots, t_{n-2}^{\prime}\right)\right| \leqq K \sum_{\nu=1}\left|t_{\nu}-t^{\prime}{ }_{\nu}\right|
$$

3) Osgood: Lehrbuch d. Funktionentheorie $\mathrm{II}_{1}$. p. 191

4) Bochner: Functions of several complex variables (1936) p. 194. Lemma, 
where $K$ is a constant and $(t),\left(t^{\prime}\right)$ are any two roints of $\Delta$. Let

$$
\eta(P)=\int_{\Delta} \ldots \int \frac{d i_{1} \ldots d t_{n-2}}{\overline{P Q^{n-2}}}
$$

where $P=\left(x_{1}, \ldots, x_{n}\right)$ is a variable point in the space and $Q=(t)=\left(\varphi_{1}(t), \ldots\right.$ $\left.\ldots, \varphi_{n}(t)\right)$ is a point of $E$, then $v(P)$ is barmonic outside of $E$. We will prove :

Lemma 3 lim $v(P)=+\infty$, when $P$ iends to any point of $E$ :

Proof. In the proof, we denote constants by $K_{1}, K_{2}, \ldots \ldots$

Let $Q_{0}=\left(t^{0}\right)$ be any point of $E$, then by (2),

$$
\overline{P Q}^{2} \geqq\left(\bar{P} Q_{0}+\overline{Q_{0} Q}\right)^{2} \geqq=K_{1}\left(r^{2}+\sum_{\nu=1}^{n-2}\left(t_{\nu}-t_{\nu}^{0}\right)^{2}\right)
$$

where $r=\overline{P Q_{0}}$.

By putting $r \tau_{2}=t_{2}-t_{\nu}{ }^{0}$, we have

$$
v^{\prime}(P) \geqq K_{2} \int_{\sum_{\nu=1}^{n-2} \tau_{\nu}^{2} \leqq-\frac{\delta^{2}}{r^{2}}} \frac{d_{\tau_{1}} \ldots \ldots d \tau_{n-2}}{\left(1+\sum_{\nu=1}^{n-\tau_{\nu}^{2}} \tau_{\nu}^{2}\right)^{n-2}}
$$

where we take $o$ so small that $\sum_{\nu=1}^{n-2}\left(i_{\nu}-t_{\nu}{ }^{0}\right)^{2} \leqq \delta^{2}$ is contained in $\Delta$.

Let $\sum_{\nu=1}^{n-2} \tau_{\nu}^{2}=i^{2}, \tau_{\nu}=\alpha_{\nu i} \rho$, then it is easily seen thrit

$$
v(P) \geq K_{3} \int_{1}^{\delta} \frac{\rho^{n-3} d \rho}{\left(1+\rho^{2}\right)^{\frac{n-2}{2}}} \geq K_{4} \int_{1}^{\delta} \frac{d \rho}{\rho^{\prime}}=K_{4} \log \frac{\delta}{r},
$$

so that $v(P) \rightarrow+\infty$, for $P \rightarrow Q_{0}$, q. e. d.

From Theorem 2 we have

Theorem 5. Let $E$ satisfy the condition of Lemma 3 and $u(P)$ be a bounded harmonic funct.on $u$ a neighbourhood of $E$, then $u(P)$ is harmonic on $E$.

Theorem 6. Let $f\left(z_{1}, \ldots, z_{n}\right)$ be a bontruld regular function of $n$ complex variables in a neighbourrhood of a $(2 n-2)$-dimensional manifold $E$, which satisfies the condition of Lemma 3 (zvith $2 n$ instead of $n)$, then $f\left(z_{1}, \ldots, s_{n}\right)$ is regular on $E$.

Mathematical Institute, Tokyo University. 